\title{
Photospheric Emission in Fermi GBM Gamma Ray Bursts
}

\section{Sinéad McGlynn* on behalf of the Fermi GBM collaboration}

Excellence Cluster Universe, Technische Universität München,

Boltzmannstr. 2, 85748 Garching, Germany.

E-mail: smcglynnetum.de

\begin{abstract}
We discuss the systematic time-resolved analysis of the brightest gamma-ray bursts observed by the Gamma ray Burst Monitor (GBM) on Fermi up to September 2011, where the prompt spectra show evidence for photospheric emission. These burst spectra can be fit with a combination of the Band function and a blackbody component, with the blackbody component being observed below the peak energy of the Band function. GRB 110920 displays the clearest signature of two separate emission components in the sample and is discussed in detail. Assuming a redshift of $z=2$, GRB 110920 has a bulk Lorentz factor of $\Gamma \sim 440$ and a photospheric radius of $6 \times 10^{11} \mathrm{~cm}$, in agreement with previously published estimates.
\end{abstract}

Gamma-Ray Bursts 2012 Conference-GRB2012,

May 07-11, 2012

Munich, Germany

\footnotetext{
* Speaker.
} 


\section{Introduction}

Since the launch of the Fermi Gamma-ray Space Telescope in 2008, deviations from the canonical Band function [1] at both low and high energies have been observed in gamma ray burst spectra (e.g.[2],[3],[4]). [5] suggested that GRB spectra are a superposition of two spectral components: photospheric blackbody emission and an accompanying nonthermal component. A fraction of the emission comes from the photosphere and another fraction is from dissipation in the optically thin region of the flow. According to the classical fireball model of GRBs (e.g. [6]), the relativistic outflow produced by the collapse of a massive star is initially radiation-dominated and accelerates from an initial radius $r_{0}$. Assuming that no dissipation takes place in the flow, the plasma expands adiabatically with a Lorentz factor $\eta(r) \propto r$ up to the saturation radius, $r_{\mathrm{s}}$. At this point the outflow becomes matter-dominated and coasts with a constant Lorentz factor, $\eta$. The photons are decoupled from the plasma at the edge of the photosphere, $r_{\mathrm{phb}}$, where the plasma becomes optically thin.

The energy that is dissipated in subphotospheric shocks will partly thermalise again to an extent that depends on the conditions at the dissipation site. [7] showed that the Planck function injected into the dissipation region is modified to a varying extent depending on the dissipation fractions and the optical depth. In the case of low dissipation, the Planck spectrum can retain its original shape. Regardless of the exact nature of the dissipation process, the electrons are expected to cool rapidly on a time-scale much shorter than the dynamical time, provided that (i) the dissipation occurs below or not too high above the photosphere and (ii) that the energy that is being dissipated is not much larger than the energy density in the photosphere. Simulations of subphotospheric shocks and their effect on the emitted photospheric photon energies [8] show that dissipation occurring at a low optical depth only marginally affects the emitted photospheric emission.

From the observed physical quantities such as thermal flux, $F_{\mathrm{BB}}$, temperature $T_{\mathrm{ob}}$, normalisation of the thermal component, $\mathscr{R}$, ratio between total fireball energy and the energy emitted in gamma rays, $Y_{0}$, the dynamics of the flow, such as the bulk Lorentz factor, $\eta$, the photospheric radius, $r_{\mathrm{phb}}$, and the initial radius of the fireball, $r_{0}$ can be derived under certain assumptions [9].

\section{Sample}

We studied the sample of bright and hard GRBs presented in [10] and updated the sample to all bright and hard long GRBs observed to the end of September 2011. All of the bursts in the sample are observed by at least one BGO detector. This resulted in 64 bursts whose spectra were split into time-resolved bins by ensuring a minimum signal to noise ratio of 15 in the detector with the smallest off-axis angle relative to the burst location. A further 26 were excluded due to a low number of time-resolved bins, where the evolution of the spectrum could not be examined. Of the remaining 38, 19 bursts can be fit with a combination of a Band and blackbody (BB) function. We therefore interpret the combination of the two functions as two separate components, where the blackbody corresponds to the photosphere, visible due to low dissipation in the outflow. In this work we discuss in particular the properties of GRB 110920, which displays both components throughout the time-resolved spectra [11]. 


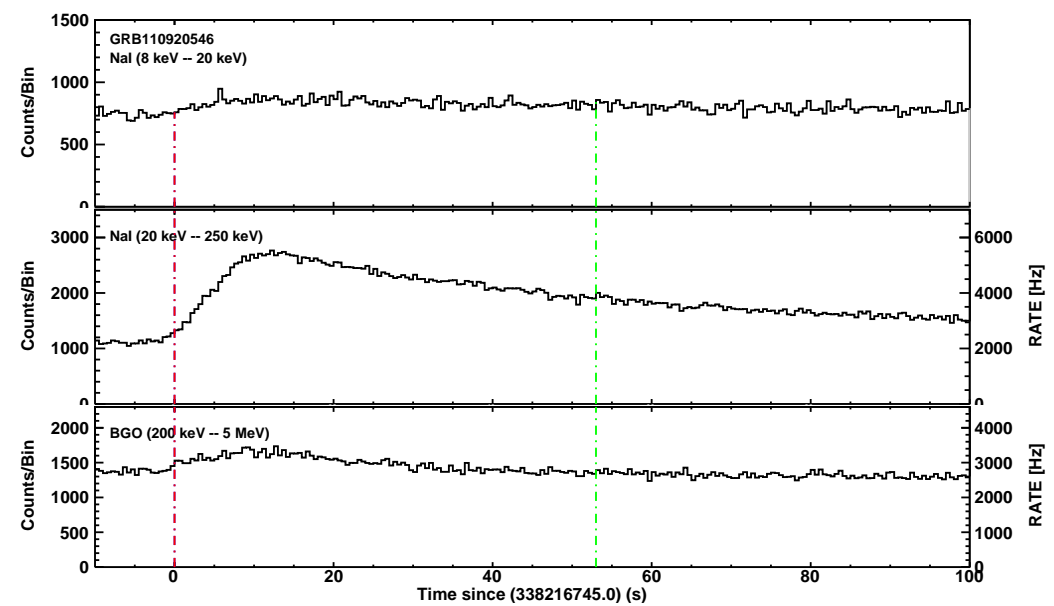

Figure 1: Lightcurve of GRB 110920. Top panel: NaI (8-20 keV). Second panel: NaI (20-250 keV). Bottom panel: BGO $0(200 \mathrm{keV}-40 \mathrm{MeV})$. The vertical lines indicate the time interval used in the spectral analysis $\left(T_{0}+0.0-T_{0}+52.7 \mathrm{~s}\right)$.
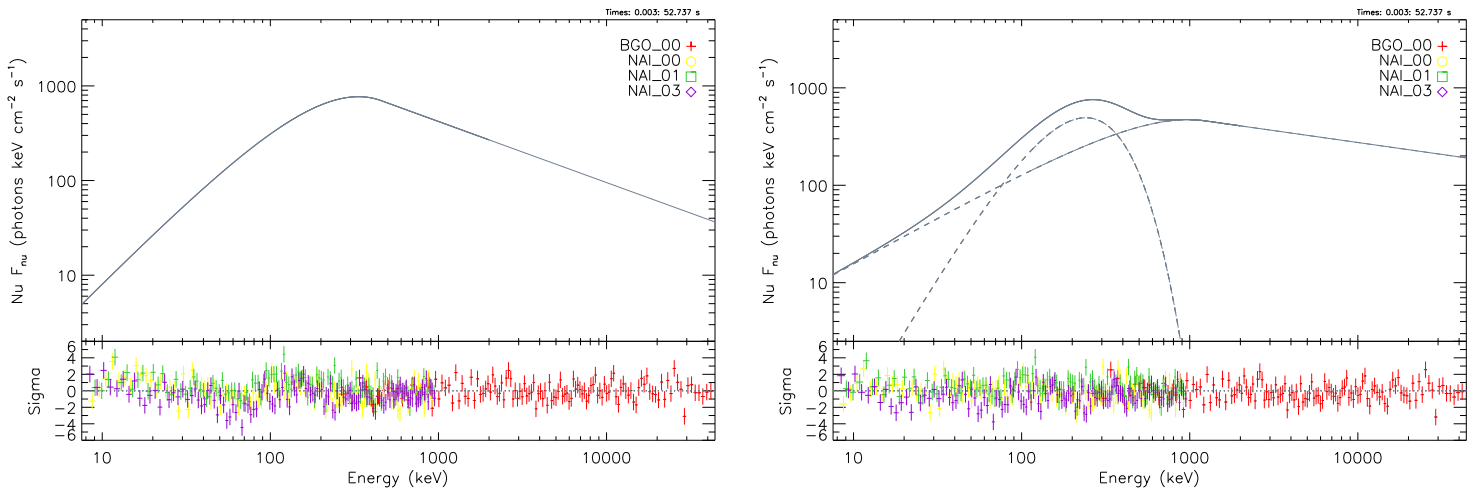

Figure 2: Time-integrated $v F_{v}$ spectra of GRB 110920 fit by the Band function (left) and Band+BB function (right).

\section{GRB 110920}

On September 20th 2011 at 13:05:43.8 (UT), GRB 110920 triggered the GBM instrument on board Fermi. The best location from GBM was at RA $=13^{\mathrm{h}} 59^{\mathrm{m}} 16.8^{\mathrm{s}}$, Dec $=-27^{\circ} 33^{\prime} 36^{\prime \prime}$, at an offaxis angle from the LAT zenith of $16^{\circ}$. The lightcurve of GRB 110920 is shown in Fig. 1 as seen by $\mathrm{NaI}$ detectors 0,1 and 3 and BGO detector 0 . The burst clearly shows a fast rise, exponential decay (FRED) structure with a $\mathrm{T}_{90}$ duration [12] of $170 \pm 17$ seconds. The bulk of the emission occurs in the 20-250 keV energy range.

The best fit Band parameters for the time interval $\left[T_{0}+0.003, T_{0}+52.737\right]$ were $\alpha=-0.20 \pm$ $0.02, \beta=-2.65_{-0.09}^{+0.07}, E_{\text {peak }}=334 \pm 5 \mathrm{keV}$ with $\mathrm{C}$-stat $=3206.5$ (485 d.o.f.), as shown in Fig. 2 (left). However, when a blackbody component was included in the fit, the C-stat is reduced to 2848.3 (483 d.o.f.) which is a difference of 358.2. The peak energy of the Band component is shifted up to $E_{\text {peak }}=978_{-121}^{+154} \mathrm{keV}$ and the temperature of the blackbody is $k T=61.3_{-0.6}^{+0.7} \mathrm{keV}$ as shown in Fig. 2 (right). The low energy index $\alpha$ becomes shallower $(\alpha=-1.05 \pm 0.04)$. 
The burst spectrum was then split into approximately 25 time bins from $T_{0}+0.003-T_{0}+$ $52.737 \mathrm{~s}$. These bins were determined by dividing the data observed in $\mathrm{NaI} 0$ into time intervals with a minimum signal-to-noise ratio of 30 counts per bin. There was a marked improvement in the $\mathrm{C}$-stat parameter $(\Delta \mathrm{C}$-stat $>10)$ when a blackbody component is added to the Band fit in 19 of the 25 bins. The blackbody flux is between $20 \%-40 \%$ of the total flux throughout the burst in the 10 $10000 \mathrm{keV}$ energy range. The blackbody temperature decreases as a power-law from $\sim 100 \mathrm{keV}$ to $\sim 40 \mathrm{keV}$ as shown in Fig. 3 (left).

\section{Interpretation}

GRB 110920 follows the general trend throughout the burst sample discussed above, where the peak energy of the Band function $E_{\text {peak }}$ is shifted to higher values due to the presence of the blackbody, creating a double-peaked spectral effect, and where $\alpha$ shifts from $\alpha \sim 0$ to $\alpha \sim-1$, becoming more consistent with synchrotron emission models.

Since there is no redshift estimate available for GRB 110920, the outflow parameters are calculated for the time resolved analysis, assuming a redshift $z=2$ (average value for Fermi GRBs), and luminosity distance, $d_{L}=4.9 \times 10^{28} \mathrm{~cm}$. The normalisation of the thermal component, which is shown in Fig. 3 (right) can be parametrized by

$$
\mathscr{R} \equiv\left(\frac{F_{\mathrm{BB}}}{\sigma T_{\mathrm{ob}}^{4}}\right)^{1 / 2}=\xi \frac{(1+z)^{2}}{d_{\mathrm{L}}} \frac{R_{\mathrm{ph}}}{\Gamma}
$$

which is proportional to the transverse size, $r_{\mathrm{phb}} / \eta$, where the baryonic photospheric radius is given by

$$
r_{p h b}=\left(L_{0} \sigma_{T}\right) /\left(8 \pi \eta^{3} m_{p} c^{3}\right)
$$

The values of the Lorentz factor $\eta$, radius of the fireball and saturation radius $r_{s}$ can thus calculated. Once $\eta$ is known, the photospheric radius $r_{\text {phb }}$ can also be determined, if $r_{\text {phb }}>r_{s}$.

The evolution of the Lorentz factor $\eta$ is shown in Fig. 4 and decreases with time from 588 to 280 , but the baryonic photosphere $r_{\text {phb }}$ remains constant at $\sim 6 \times 10^{11} \mathrm{~cm}$ throughout the burst. The average parameter values are $r_{\mathrm{phb}}=5.9 \times 10^{11} Y_{0}^{1 / 4} \mathrm{~cm} ; \eta=442 Y_{0}^{1 / 4} ; r_{0}=2 \times 10^{8} Y_{0}^{-3 / 2} \mathrm{~cm}$ and $r_{\mathrm{s}}=8.6 \times 10^{10} Y_{0}^{-5 / 4} \mathrm{~cm}$; where $Y_{0}$ is the ratio of the total fireball energy to the gamma ray energy, and is close to unity [9]. These values are close to previous estimates of the outflow parameters (e.g. [13] [4]).

\section{References}

[1] D. Band, J. Matteson, L. Ford et al., BATSE observations of gamma-ray burst spectra. I - Spectral diversity, ApJ 413 (1993) 281

[2] A. A. Abdo, M. Ackermann, M. Ajello et al., Fermi Observations of GRB 090902B: A Distinct Spectral Component in the Prompt and Delayed Emission, ApJ 706 (2009) L138

[3] M. Ackermann, M. Ajello, K. Asano et al., Detection of a Spectral Break in the Extra Hard Component of GRB 090926A, ApJ 729 (2011) 114

[4] S. Guiriec, V. Connaughton, M. Briggs et al., Detection of a Thermal Spectral Component in the Prompt Emission of GRB 100724B, ApJ 727 (2011) L33 

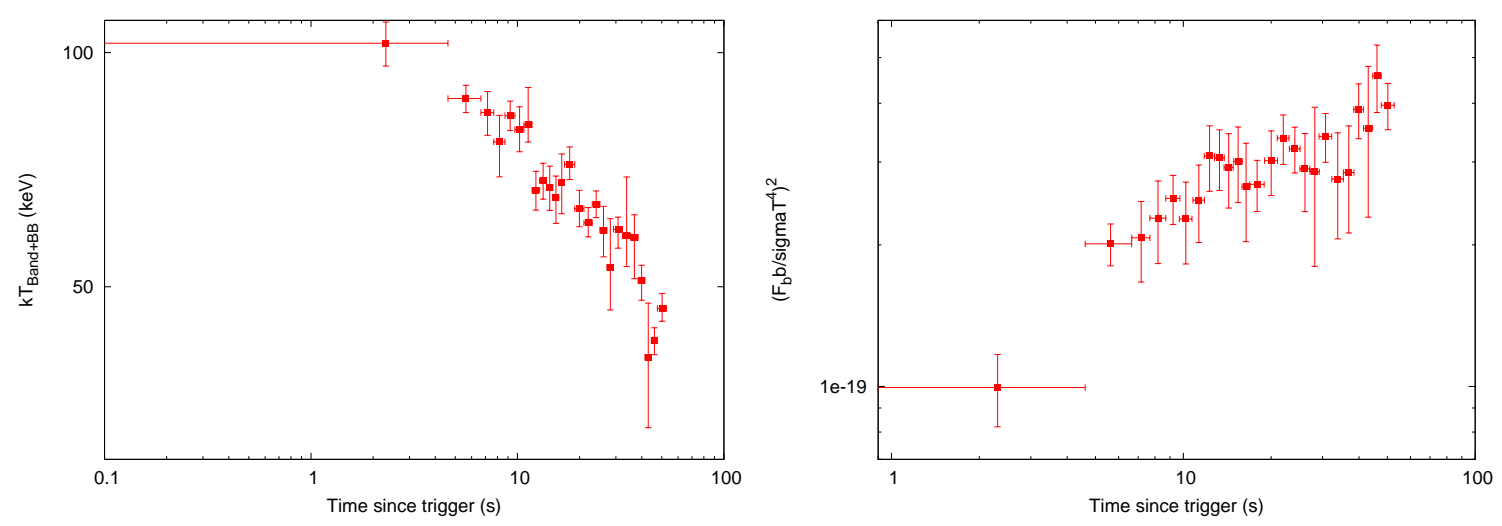

Figure 3: Evolution of the temperature (left) and normalisation parameter $\mathscr{R}$ (right).

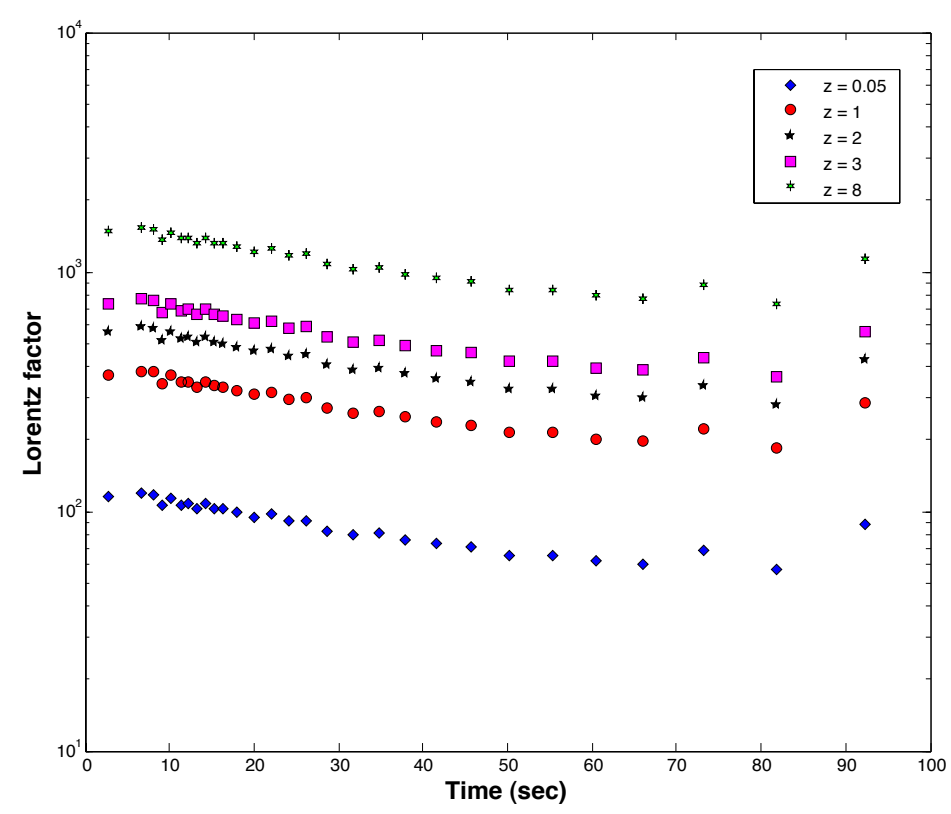

Figure 4: Evolution of $\Gamma$ throughout GRB 110920 at varying redshift.

[5] F. Ryde, The Cooling Behavior of Thermal Pulses in Gamma-Ray Bursts, ApJ 614 (2004) 827

[6] P. Mészáros, Gamma-ray bursts, Rep. Prog. Phys. 69 (2006) 2259

[7] A. Pe'er, P. Mészáros and M. Rees, The Observable Effects of a Photospheric Component on GRB and XRF Prompt Emission Spectrum, ApJ 642 (2006) 995

[8] F. Ryde, A. Pe'er, T. Nymark et al., Observational evidence of dissipative photospheres in gamma-ray bursts, MNRAS 415 (2011) 3693

[9] A. Pe'er, F. Ryde, R. A. M. J. Wijers et al., A New Method of Determining the Initial Size and Lorentz. Factor of Gamma-Ray Burst Fireballs Using a Thermal Emission Component, ApJ 664 (2007) L1

[10] E. Bissaldi, A. von Kienlin, C. Kouveliotou et al., First-year Results of Broadband Spectroscopy of the Brightest Fermi-GBM Gamma-Ray Bursts, ApJ 733 (2011) 97 
[11] S. McGlynn et al., Photospheric Emission in the Fermi Gamma ray Burst GRB 110920, in prep (2012)

[12] C. Kouveliotou, C. A. Meegan, G. J. Fishman et al., Identification of two classes of gamma-ray bursts, ApJ 413 (1993) L101

[13] J. Larsson, F. Ryde, C. Lundman et al., Spectral components in the bright, long GRB 061007: properties of the photosphere and the nature of the outflow, MNRAS 414 (2011) 2642 